\title{
BMJ Open Hyperbaric Oxygen in Lower Limb Trauma (HOLLT); protocol for a randomised controlled trial
}

\author{
Ian L Millar, ${ }^{1,2}$ Rosemary A McGinnes, ${ }^{2}$ Owen Williamson, ${ }^{2}$ Folke Lind, ${ }^{3}$ \\ Karl-Åke Jansson, ${ }^{4}$ Michal Hajek, ${ }^{5,6}$ David Smart, ${ }^{7}$ Tiago Fernandes, ${ }^{8}$ \\ Russell Miller, ${ }^{9}$ Paul Myles, ${ }^{10}$ Peter Cameron $^{2}$
}

To cite: Millar IL, McGinnes RA, Williamson 0, et al. Hyperbaric Oxygen in Lower Limb Trauma (HOLLT); protocol for a randomised controlled trial. BMJ Open 2015;5:e08381.

doi:10.1136/bmjopen-2015008381

- Prepublication history for this paper is available online. To view these files please visit the journal online (http://dx.doi.org/10.1136/ bmjopen-2015-008381).

Received 1 April 2015 Accepted 13 May 2015

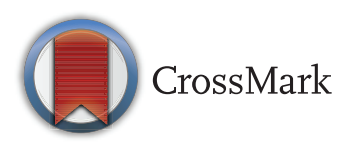

For numbered affiliations see end of article.

Correspondence to Dr lan L Millar; i.millar@alfred.org.au

\section{ABSTRACT}

Introduction: Open fractures with significant soft tissue injury are associated with high rates of complications, such as non-union, infection, chronic pain and disability. Complications often require further inpatient care, and in many cases, multiple operations and prolonged rehabilitation. Use of hyperbaric oxygen therapy as an adjunct to standard orthopaedic trauma care has the potential to reduce the complications of musculoskeletal injury and thus improve outcomes. Two previous randomised trials have suggested some positive effect, but neither functional measures nor long-term outcomes were reported.

Methods and analysis: An international, multicentre, randomised, open-label, clinical trial. Patients with trauma with an acute open fracture of the tibia with severe soft tissue injury (Gustilo grade 3) and high risk of injury-related complications were recruited from participating major trauma hospitals with hyperbaric facilities. Patients were enrolled with the expectation of commencing 12 sessions of hyperbaric oxygen therapy within $48 \mathrm{~h}$ of injury. The primary outcome measure is the incidence of acute complications of the open fracture wound at 14 days. Other short-term outcome measures include amputation, need for fasciotomy, time until wound closure, breakdown of closed wounds, time until definitive orthopaedic fixation, number of operative procedures, intensive care stay and hospital stay. Long-term follow-up will continue for 2 years postinjury.

Ethics and dissemination: Ethics approval was given by The Alfred Health Human Ethics Committee (206/04) and the Monash University Human Research Ethics Committee (CF07/4208). Approval was also obtained from the institutional research ethics committee at each participating site. This study will make a significant contribution to the trauma literature and should answer the question of whether hyperbaric oxygen therapy can significantly improve outcomes in severe lower limb trauma. Collective study results will be published in international journals and presented at relevant conferences.

Trial registration number: Clinicaltrials.gov: NCT00264511; Australian New Zealand Clinical Trials Registry (ANZCTR): ACTRN12607000559415.

\section{Strengths and limitations of this study}

- A prospective, randomised study with blinded outcome scoring.

- Open-label design with standard trauma care as the control.

- Multiple centres involved from a variety of countries.

- Very slow recruitment.

- Target enrolment reduced from initial plans.

\section{INTRODUCTION}

Orthopaedic trauma is common and responsible for a high level of social and personal cost as well as increased hospital resource use. ${ }^{12}$ Fractures are the most common of all forms of hospitalised trauma, accounting for $36 \%$ of all injury hospitalisations in Australia. ${ }^{3}$ The detailed epidemiology of orthopaedic injury is complex, with widely varying incidences reported for various fracture types, with some real geographical differences as well as methodological reasons for this. While the majority of fractures are closed, open fractures are of particular importance as they present more complex management challenges, and are associated with higher risks of complications. Open fractures are also more frequently associated with higher energy trauma and more severe soft tissue injury. The overall incidence of open long bone fractures has been reported as $11.5 / 100000$ persons per year with the majority of these being severe tibial fractures. ${ }^{4}$ Tibial shaft fractures are one of the most common long bone fractures. The incidence of these fractures seems to have fallen somewhat over recent years, but there are still around 14-17 fractures reported per 100000 per year in developed nations, ${ }^{5} 6$ with around one in five of these being open. $^{7}$ 
Open tibial fractures with significant soft tissue injury are associated with particularly high rates of complications. Lengthy healing time, delayed or non-union, infection, wound breakdown, compartment syndrome and osteomyelitis are frequently reported. ${ }^{8}{ }^{9}$ Many of these problems require extended inpatient care, and often multiple operations with prolonged rehabilitation requirements, and high associated costs. ${ }^{9}{ }^{10}$ In addition to clinical outcomes, disability and inability to return to work are common, along with chronic pain. These adverse outcomes often persist for 12 months or more following orthopaedic trauma. ${ }^{11-14}$

Hyperbaric oxygen (HBO) has the potential to reduce the complications of orthopaedic injury and thus improve outcomes. HBO has been used as a treatment for crush injury and compartment syndromes in a limited number of centres around the world, and is often recommended in hyperbaric medicine-specific literature. ${ }^{15-17}$ The basis for this and clinical experience published to date have been systematically reviewed recently. ${ }^{18-22}$ While individual studies show promising results, the consensus is that further clinical investigation is required before $\mathrm{HBO}$ can be endorsed strongly enough for changes to be made to existing trauma systems and trauma centre infrastructure.

A large variety of animal models of soft tissue flap ischaemia have demonstrated the ability of HBO to preserve acutely ischaemic tissue and improve flap survival, while others have demonstrated substantially accelerated and improved quality of healing of muscle, tendon, peripheral nerve and bone. ${ }^{15} 16$ 23-29 $\mathrm{HBO}$ can significantly reduce post-trauma oedema, moderate inflammatory processes and upregulate various endogeneous antioxidant systems. HBO during or immediately after ischaemia can inhibit ischaemia-reperfusion injury via mechanisms that include inhibition of 32 integrin-mediated neutrophil adhesion during reflow. This effect does not inhibit neutrophil-mediated bacterial killing which is, by contrast, enhanced. ${ }^{30}$ Bacterial killing is known to be highly oxygen dependent, and $\mathrm{HBO}$ can maximise endogeneous and pharmacological antibacterial activity via neutrophils as well as by directly inhibiting anaerobes and augmenting the action of antibiotics, such as aminoglycosides which are impaired in hypoxic conditions. HBO also accelerates angiogenesis and fibroblast function to improve healing of problem wounds. ${ }^{15}$ All these actions are attractive in the setting of orthopaedic and soft tissue trauma. ${ }^{31}$

In humans, the effect of HBO on tibial shaft fractures fixed with intramedullary nailing has been studied with a randomised controlled trial. A positive effect was observed on arterial flow and local skin perfusion measures, suggesting improved soft tissue perfusion and oxygenation in the postoperative days. ${ }^{32} \mathrm{~A}$ second randomised controlled trial looking at the effect of HBO therapy in the management of severe limb injury involving crush injuries also provides evidence for some efficacy of HBO in severe limb injury. Thirty-six patients with Gustilo Grade 2 or 3 crush injuries, not limited to tibial fracture, were assigned in a blinded, randomised fashion to receive $\mathrm{HBO}$ or placebo within $24 \mathrm{~h}$ of surgery and, subsequently, twice daily over 6 days. Seventeen patients in the HBO group versus 10 in the placebo group achieved complete healing $(p<0.01)$, and further surgery was required in one of the HBO group versus six in the placebo group $(\mathrm{p}<0.05) .{ }^{33}$ Neither of these studies reported on subsequent outcomes following these encouraging acute effects.

A randomised, sham, controlled, pilot study of HBO in Gustilo 3b and 3c tibial shaft fractures was conducted at The Alfred Hyperbaric Service in Melbourne in 2001/2002. Seventeen patients were entered into this study, and many logistic and practical issues involved in the management of severe trauma patients were identified and resolved. The study numbers were insufficient to produce any statistically significant outcome differences, but this pilot and our ongoing registry activities gave us injury incidence data on which to base predictions of enrolment rates for future studies, as well as informing our later selection of protocol and data points for the Hyperbaric Oxygen in Lower Limb Trauma (HOLLT) study.

The HOLLT project started with the forming of an international steering group which reached agreement upon many of the key study design features and the practicalities of conducting a multicentre randomised controlled trial. More than 50 sites expressed interest in participating, although only a small proportion was ultimately able to join the study. The detailed study data set was then developed by a Monash University working group with input from the initial group of international collaborators.

In designing this study, we concluded that severe lower leg injury was the most suitable target for a randomised controlled trial of the effects of $\mathrm{HBO}$ on acute musculoskeletal injury. Severe open fractures of the tibia are associated with high rates of complications such as osteomyelitis and non-union. ${ }^{8}$ Infection rates for complex fractures with severe soft tissue injury graded as Gustilo $3 \mathrm{~b}$ have been reported to be in the range of $25-50 \%,{ }^{34-36}$ and with amputation rates in the range of $4-16 \%{ }^{1}$ Gustilo 3c tibial fractures (vascular injury present) are associated with major complication rates of up to $100 \%$ and amputation rates of $60 \%$ or more. ${ }^{37-41}$ The complications of wound breakdown and infection are also common after high-energy pilon fractures. ${ }^{42}$ We hypothesise that by reducing soft tissue complications, early treatment with $\mathrm{HBO}$ will improve outcomes.

\section{Objectives}

To investigate the study hypotheses that a course of $\mathrm{HBO}$ will reduce the rate of short-term and long-term complications of lower limb injury involving tibial fracture with severe soft tissue injury, and that this will be associated with measurably improved functional and quality of life outcomes for patients. 
Note: At the time of finalising the manuscript for this paper, enrolments had closed, but data collection was ongoing and results had not been analysed. The protocol that follows has been edited to suit publication, but accurately reflects the details included in the HOLLT Protocol 8.1 dated 27 May 2011 and the Study Manual V.4.0 dated 18 June 2010 which are the current documents being followed by all collaborators in this project. These documents, as well as previous versions, are held on file at Monash University, and are available from the Principal Investigator upon request.

\section{METHODS AND ANALYSIS}

\section{Trial design}

This trial was designed as an international, multicentre, randomised, clinical trial of a therapeutic intervention (HBO therapy) versus no intervention, in addition to standard clinical care. The surgical team initially operating upon the patient was blinded until after initial surgery was completed. Once consent was obtained and the patient was enrolled, allocation was made on a 1:1 randomised basis to either the hyperbaric treatment or to the control group. From this point onwards, the treating team, data collectors and all subsequent care providers have not been blinded. Outcome assessors reviewing data in order to determine derived, scored or arbitrated outcomes are all blinded to patient identity, intervention group allocation and the collaborating site managing the patients whose data they are reviewing.

\section{Setting and participants}

Adult trauma patients with severe lower leg injuries admitted to participating major trauma hospitals with HBO facilities.

\section{Inclusion criteria}

- Persons who have suffered an acute open fracture of the tibia with severe soft tissue injury and a high risk of injury-related complications as judged by an assessment of the injury, the injury mechanism(s) and the host status. Most were expected to be graded as Gustilo Grade 3.

- Minimum age 18 years (or older where local consent procedures require this).

- Enrolment within $48 \mathrm{~h}$ of injury with the expectation of start of HBO therapy as soon as practical.

Note: the enrolment window was initially set at $24 \mathrm{~h}$, but was increased to $48 \mathrm{~h}$ early on in the study. The decision was based upon logistic difficulties of early enrolment in many cases, and the consensus clinical experience of the researchers who felt that $\mathrm{HBO}$ could still offer significant effects even if started up to $48 \mathrm{~h}$.

- Permission to enrol patient granted by the trauma team managing the patient.

- Consent from patient or person responsible, as determined by local law.

\section{Exclusion criteria}

- Other injuries, instability or resuscitation/intensive care requirements judged by the trauma surgeon, or intensive care specialist, to be incompatible with safe HBO therapy.

- Patients suffering advanced medical conditions where the severity of the condition was such that the patient was not expected to survive for the follow-up period.

- Patients suffering psychiatric disorders, or having incurred the injury through a self-harm or suicide attempt.

- End-stage peripheral vascular disease, or other limb problems which would be expected to progress to amputation, ulceration or gangrene over the follow-up period, had the injury not happened.

Note: Vascular disease or diabetic neuropathy affecting the injured leg was acceptable, and in some cases may be a risk factor leading to the fracture being considered severe, provided the disease severity is not such that early amputation would be the likely or preferred treatment for the injury.

- Contraindications to hyperbaric therapy including pregnancy.

\section{Interventions}

Participants were randomised 1:1 to either the $\mathrm{HBO}$ or No HBO group. The HBO group received $\mathrm{HBO}$ therapy in addition to standard trauma care, while the No HBO (control) group received standard trauma care only. Those who chose not to participate in this research trial received the same medical and nursing care that would normally be provided for trauma victims presenting to the treating hospital.

For patients in the HBO treatment group, the aim was for them to receive a total of $12 \mathrm{HBO}$ sessions over approximately 8 days, unless circumstances prevented this, for instance, where the patient was progressing well enough to be discharged from hospital. HBO treatment was provided using pressure/time schedules selected from existing protocols by each participating centre, aimed at delivering a dose approximately bioequivalent to $90 \mathrm{~min}$ of oxygen breathing at 2.4 atmospheres absolute pressure. Treatments were delivered twice daily for the first 3 days except where competing care requirements made this logistically impossible. Scheduling variability was allowed with respect to the timing and profile of each session in accordance with the logistic practicalities of operating a hospital-based hyperbaric medicine centre. Overall, HBO patients received therapy as would occur in normal clinical practice outside of a research study, and in many cases were treated alongside patients being treated for other indications. Less than 12 HBOT sessions were accepted as a full course if the patient was able to be discharged from the acute hospital before the planned 12 HBO sessions could be delivered. For outcome analysis by therapy delivered, the successful provision of $6 \mathrm{HBO}$ sessions was arbitrarily chosen as 
qualifying the patient for analysis as having received a successful course of HBO therapy.

\section{Primary outcome}

The primary outcome measure for this study is the incidence of acute fracture wound complications after injury. This is a composite measure defined as the occurrence within 2 weeks of injury of one or both of significant soft tissue necrosis developing after the initial surgery, or significant wound infection.

- Significant soft tissue necrosis will be determined based upon the amount of tissue surgically debrided after the initial surgery. Minimal trimming of skin edges removing no more than a few millimetres of tissue to clean the surface of a wound which was not obviously necrotic will be considered normal surgical practice and will not be scored as indicating 'significant necrosis'.

- Wound infection occurring during the acute phase will be assessed via review of data based upon those used in the USA Centre for Disease Control guidelines for assessing surgical wound infections. ${ }^{43}$

- The final adjudication on any cases where the presence of infection or necrosis is equivocal will be determined via review of available clinical data, photos and X-rays by clinical adjudicators not involved in the patient's care and blinded to the identity and location of the patient, and the allocation to $\mathrm{HBO}$ or no-HBO group.

- The target time point for recording of this primary outcome data was approximately day 14 , or when the patient was discharged from hospital where discharge occurred earlier. In cases where early discharge occurred, follow-up data is pursued to identify any readmissions for complications.

\section{Secondary outcomes}

Early secondary outcome measures: The day 14 and hospital discharge data set includes the following data: date, time and nature of all operative procedures, the undertaking of fasciotomy or amputation, time until bone coverage and until skin closure and methods used, breakdown of closed wounds where this occurred, nature and timing of orthopaedic fixation(s), length of stay in ICU, length of stay in the acute hospital, and for patients receiving HBO therapy, the details of HBO sessions provided including any side effects or complications.

Late secondary outcome measures: The late secondary outcomes are derived from clinical, radiological and questionnaire data collected at reviews scheduled for approximately 3, 6, 9, 12, 18 and 24 months. From this data, assessments will be made for the occurrence of soft tissue infection, osteomyelitis, any other wound complications, whether the patient is able to bear weight, and the number and nature of any other surgical procedures that have been required.

The primary indicator that delayed or non-union has occurred is the recording of a bone graft having been undertaken, or use of non-standard adjuncts such as electrical stimulation. The indicators for late deep infection include prolonged courses of antibiotics and/or debridement surgery. For any such outcomes that may be unclear, blinded data and imaging review will be performed by two independent orthopaedic surgeons who will adjudicate the final recorded outcome.

Functional and quality-of-life outcome measures include the return-to-work component of the Sickness Impact Profile, a simple comparative global disability scale and a visual analogue pain scale. At 12 months and 2 years, a language-appropriate version of the SF36 questionnaire, and the lower limb injury component of the Short Musculoskeletal Functional Assessment are recorded.

The scoring of radiological union is based upon blinded review of X-rays taken at 3, 6, 9, and 12 months, and at 18 months and 2 years in cases not united at 12 months. Review and scoring of radiographs will be performed by assessors who are blinded to the trial group allocation and the time at which the radiographs were taken postinjury. Interobserver and intraobserver consistency will be determined.

\section{Sample size and power calculation}

At the start of this trial, a sample size of 250 persons was selected to provide $80 \%$ power to detect a reduction in the incidence of a defined set of acute complications from $30 \%$ to $15 \%$ at $\mathrm{p}=0.05$. Selection of $30 \%$ as the estimated control complication rate was based upon our pilot study data and is mid-range with respect to published complication rates for the injuries targeted.

The sample size was subsequently revised to 120 patients following a review of the viability of the HOLLT Study triggered by slow recruitment. An assessment of the blinded outcome data available for the first 40 patients showed an event rate of $51.2 \%$ which was higher than was expected, and a doubling of the event rate upon which the study was powered. The Data Safety and Monitoring Committee provided support to the study's Project Management Group for this change to occur.

\section{Recruitment}

Recruitment methodology varied between participating institutions, as proved practicable. Identification of patients variously involved the emergency department and/or trauma teams, trauma and orthopaedic surgeons, anaesthetic staff and others. Where available, computerised admission and operating theatre data were reviewed. The aim was for patients to be identified upon presentation or during initial trauma surgery, but on occasions, patients were identified from secondary sources after the initial surgery had been completed. The aim was for enrolment to be pursued at the earliest opportunity so that, if an enrolled patient were allocated to receive $\mathrm{HBO}$, then the first session would be able to commence as soon as possible after completion of initial surgery. Figure 1 shows the study timeline. 


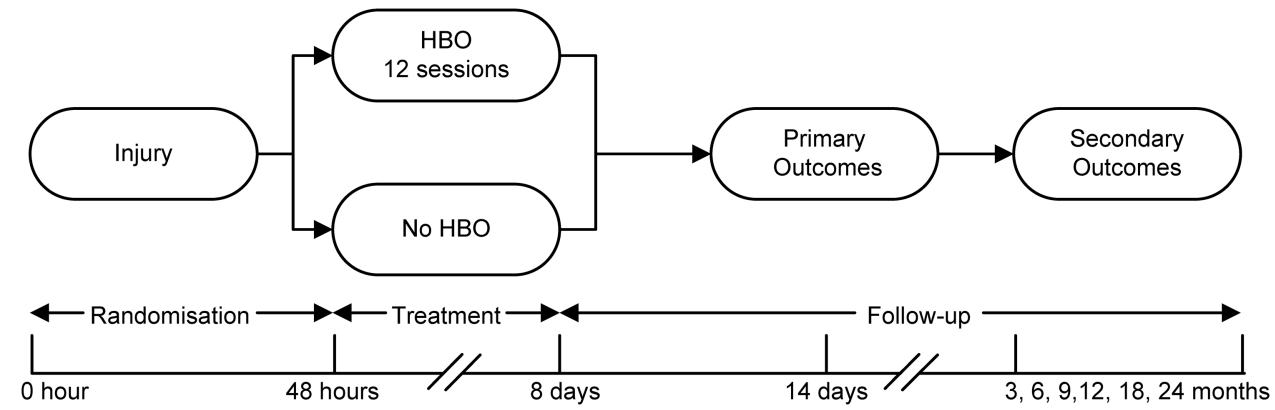

Figure 1 Timeline for Hyperbaric Oxygen in Lower Limb Trauma (HOLLT) study participants. HBO, Hyperbaric Oxygen.

\section{Randomisation and blinding}

Participating patients were allocated a randomly generated identification code, and were assigned to the HBO intervention or the control group via a computerised block randomisation schedule, stratified by site to reduce the risk of disproportionate allocation to one group or the other at any one site. Confirmation of enrolment, allocation of a study identification number and allocation to intervention group were obtained via secure $\log$ on to a web-based randomisation service based at the Coordinating Centre at the Department of Epidemiology and Preventive Medicine (DEPM), Monash University, thus ensuring allocation concealment. The computer programme providing this service is maintained by an information technology specialist independent of the research team, and investigators cannot make changes. All interactions with this study enrolment, allocation and 'Web Entry Data System' (WEDS) have been and continue to be logged and timestamped. Each investigator or research nurse enrolled patients onto the WEDS and obtained intervention group allocation only after informed, written consent was obtained, which many times occurred after the first acute trauma surgery procedures. The surgeon initially operating on the patient was in all cases kept blind to the intervention group allocation until the initial surgical procedure was completed. Investigators, clinical teams and patients in this study were also blinded to the therapeutic intervention until completion of the first surgical intervention.

The scoring of outcomes derived from primary data, and the adjudication of any uncertain outcomes have been deferred until enrolments were complete and the relevant data collected. Such assessments will be made by clinicians not involved in the care of the patient and unaware of the intervention group allocation. These reviewers and adjudicators will be supplied with data and/or digitised images and X-rays, deidentified such that there is no indication of site or intervention allocation, so as to minimise risk of bias.

\section{Data collection and management}

An initial data collection form (DCF) was completed for each study participant summarising all clinical screening and study data. Patients are only referred to in the DCF by their patient number and initials in order to retain patient confidentiality.

Data is transmitted to the Coordinating Centre using a secure web-based electronic data entry system with automatic data integrity checks built in. Photographs and $\mathrm{X}$-rays are emailed as digital images. The Coordinating Centre holds only material which is deidentified with respect to identity of the patient, other than the site where the data originated, and basic demographic data including age and occupation. The keys to connect any individual record with a particular patient are held by each site for their own patients. Detailed data management procedures can be found in the HOLLT Manual of Operations V.4 dated 18 June 2010.

\section{Statistical analysis}

Primary analysis will be by intention to treat. Analysis will also be conducted according to treatment actually received with a minimum of six completed HBOT sessions to be considered as a therapeutic course of HBO therapy. A large data set is being collected, including injury mechanism, orthopaedic and soft tissue injury characteristics, preinjury health status, other injuries, surgical and ancillary therapies utilised, as well as data on the hyperbaric therapy, and all the outcome measures detailed above.

The outcomes for this study include a mixture of categorical and continuous variables. Categorical data will initially be analysed using $\chi^{2}$ tests for equal proportion, and then analysed in a multivariate logistic regression adjusting for any baseline imbalances that may occur between groups or for any other potentially confounding variables.

In the case of continuous variables, if the variable is found to follow a normal distribution, or can be normalised using transformation, then univariate analysis will be conducted using t tests and linear regression, and a multiple regression model will then be constructed. If however, it is found that the end point is not normally distributed, (as expected for most variables in this study) univariate analysis will be conducted using nonparametric statistics. Multivariate models will then be constructed using binomial outcomes. 


\section{Monitoring}

Provision was made for monitoring via site visits and via specific data integrity reviews via email or telecommunication. A number of such monitoring visits were undertaken by representatives of the Project Management Group and the International Steering Group during the recruitment period. The purpose of these visits was to confirm confidence that the study has been conducted according to the protocol. There were also a large number of automatic validation and data integrity checks built into the WEDS database.

\section{Adverse events}

Each site investigator and the Principal Investigator are responsible for the detection and documentation of events meeting the criteria and definition of an adverse event (AE) or a serious adverse event (SAE). It is a requirement that all SAEs thought to be related to participation in the study are reported to the Principal Investigator, or designate, within $48 \mathrm{~h}$ of knowledge of the event occurring.

\section{Trial organisation}

International Steering Group

An International Steering Group was established as part of the process of moving this study from concept to reality. The Group determined a number of the primary design features of the study, including advising that the study intervention needed to be delivered on an 'open label' basis with the No HBO group not receiving a sham HBO session. The Group participated in identification and recruitment of collaborating sites and provided input into the data set before disbanding once the study was fully underway.

\section{Project Management Group}

The Project Management Group includes Monash University and Alfred Hospital-based investigators and project management personnel, with the project's European Coordinator seconded to this group as required. The Project Management Group takes responsibility for the overall design of the study and for ensuring that the execution and management of the project are of the highest quality. Group members from Monash University and the Karolinska Institute have extensive knowledge of clinical trial design and data analysis techniques. The Project Management Group includes one orthopaedic surgeon, whose input is supplemented by two additional orthopaedic trauma surgeons with research expertise based at The Alfred Hospital and the Karolinska Hospital, respectively. The Project Management Group has met over the duration of the study in order to review the conduct of the study, including rates of accrual and AEs.

\section{Data Safety and Monitoring Committee}

An independent Data Safety and Monitoring Committee (DSMC) was established comprising experts in clinical trials, biostatistics and hyperbaric medicine. The DSMC has reviewed all study details and oversees the conduct of the study. Specifically, it has provided oversight as to whether the study was on track to reach its targets within a reasonable time frame, reviewed protocol compliance, logistics and HBO-related complication rates, and was tasked with ensuring that specific events or developments did not justify halting the study. The DSMC was made responsible for authorising and reviewing any interim analyses. The only such interim review that has been undertaken was the early 'futility analysis' that occurred when initial enrolment rates were slow. This led to revision of the enrolment target, but unblinding of data was not required.

\section{Study Coordinating Centre}

The Study Coordinating Centre is located at Monash University, DEPM, Melbourne, Australia. It has been responsible for the overall coordination of the study, including the details of protocol development, design of case report forms and the WEDS, operations manuals, study materials and data management. The Study Coordinating Centre has been and remains responsible for monitoring the study execution, particularly with respect to the methodological aspects, ensuring adherence to the study protocol by the collaborating sites and study personnel involved and preparation of summary information and reports. The Monash Coordinating Centre will be responsible for the final analysis of the data set in consultation with the investigator group.

\section{ETHICS AND DISSEMINATION}

\section{Ethics Committee, or Institutional Review Board approval}

The original HOLLT study protocol and all amendments received approval from The Alfred Health Human Ethics Committee (206/04) and the Monash University Human Research Ethics Committee (CF07/4208). Approval was also obtained from the institutional research ethics committee at each participating site. Human Research Ethics approvals have been kept current for the duration of the study.

\section{Ethical conduct of the study}

This study is being conducted according to the Declaration of Helsinki, the Australian NHMRC National Statement on Ethical Conduct in Human Research $(2007),{ }^{44}$ the Notes for Guidance on Good Clinical Practice as adopted by the Australian Therapeutic Goods Administration (2000) $(\mathrm{CPMP} / \mathrm{ICH} / 135 / 95),{ }^{45}$ and the ICH GCP Guidelines.

\section{Patient consent}

The informed consent of each patient participating in the study was obtained using the locally approved and language-appropriate version of the Patient Information and Consent Form. Where local procedures allow, patients with diminished capacity were able to be enrolled with the consent or acknowledgment of a 
person responsible, such as a close relative, with confirmation consent sought from the patient once the patient became competent. It was a requirement that before enrolment into the study, each prospective candidate (or person responsible, where applicable) needed to be given a full explanation of the nature and purpose of the study, all potential risks and benefits, and a copy of the Patient Information Sheet to review. Once the essential study information had been provided, and the relevant site investigator was assured that the potential participant understood the implications of participating in the study, consent to participate in the study was recorded by the signing of the appropriate Patient Information and Consent Form (PICF). The PICF required that the signing be witnessed by a third party and appropriately dated. Notation that written informed consent had been obtained was recorded onto the patient's DCF, and confirmation of consent was required by the WEDS before an enrolment could be confirmed, and the study group allocation provided. The completed consent forms are retained by site investigators as required by their local Human Research Ethics Committee, and a copy of the PICF was required to be provided by the investigator to the patient or their delegate.

\section{Confidentiality}

The confidentiality of participant records will be protected, respecting the privacy and confidentiality rules of the applicable regulatory authorities. To comply with the Health Privacy Principles (Summary) Victoria Health Records Act 2001, and relevant legislation/guidelines for each participating country/state, there will be no transborder flow of identified data, and it is expected that there will be no flow of identified data out of any participating hospital unless allowed for by a separate and approved registry arrangement, research protocol or local legislation.

At the time of enrolment, each patient was assigned a unique study identification number. This is the only direct identifier included on the electronic DCF. Each site holds the link connecting its patient data to the DCF and to local hospital records. It has been, and is a requirement, that patient names on X-rays, wound photos or other source documents are obliterated before transmission to the Study Coordinating Centre. At each study site, all records are to remain secure, either in a locked filing cabinet in a secure area, or as password-protected computer files, as appropriate.

\section{Dissemination policy}

Results from the primary outcome analysis will be submitted for publication in a major international journal as soon as possible after collecting the relevant data and undertaking the appropriate evaluations. Subsequent papers will be published, as secondary outcome data from longer term follow-up becomes available. Collaborating investigators may make local presentations on the study and are encouraged to refer to the study in presentations and publications to broader audiences, but should not publish details of the study or its outcomes without the agreement of the HOLLT Principal Investigator.

The final data set will be held by the Monash Coordinating Centre. Study data obtained from each collaborating site will be made available to that site, on request, and can be compared with grouped data but not the data from any other individual identifiable site. A detailed data set will have been collected, and after primary publication of the study it is intended that collaborators be allowed to access the (site deidentified) data set to conduct their own additional research analyses should they wish to do this.

The trial protocol and manual may be made available to other researchers upon request. An anonymised data set will be made available on the Dryad Digital Repository. There are no plans to proactively disseminate results to individual participants.

\section{DISCUSSION}

When there is no consensus within the expert clinical community about the comparative merits of the alternatives to be tested, a state of 'clinical equipoise' exists. ${ }^{46}$ While individual studies of $\mathrm{HBO}$ in the treatment of crush injury and compartment syndromes show promising results, the quality of this evidence is very limited. ${ }^{47} 48$ The use of HBO for trauma is not widespread and there is no consensus as to whether HBO adds value to the management of acute musculoskeletal trauma. In the light of this, and after careful review of the evidence, our group felt that clinical equipoise exists, and that a suitable trial was therefore essential. In developing plans for the HOLLT Study, we discovered that HBO therapy was considered 'standard care' at a number of high-profile sites, with some key clinician's views sufficiently fixed that the conduct of the trial was impossible at these institutions. This significantly limited the potential pool of sites that could potentially collaborate, but we did not feel there were sufficient such sites to destroy the overall state of clinical equipoise. A much larger number of sites use $\mathrm{HBO}$ for trauma on an irregular basis, or use HBO for late complications, but not during the acute postinjury phase.

In addition to having a hyperbaric service capable of treating the patients involved in the study, sites with the potential to participate required an active trauma service with appropriate relationships between relevant departments. There are a limited number of such sites worldwide. Even at collaborating sites, we found that the views of individual physicians vary widely, which compromised recruitment but confirmed our belief that this study would have importance in informing future reorganisations of trauma systems.

In selecting sites for collaboration, we excluded several sites where it is standard practice to manage open 
fractures with soft tissue deficits by immediate microsurgical soft tissue transfer. Sites selected as collaborators generally follow the 'damage control' surgical approach in which surgeons initially use temporary external fixation and leave wounds open followed by delayed definitive internal fixation and wound closure several days later. Vacuum dressings are often used to temporarily manage tissue deficits pending closure. This approach may reduce systemic complications of trauma, and it offers improved logistic opportunities for $\mathrm{HBO}$ to be applied after initial surgery and in the interval leading to definitive wound closure and, where definitive orthopaedic fixation is not performed initially, in the interval preceding definitive fixation. ${ }^{49} 50$

Studies of adjuvant therapies such as this one, are only applicable if functional limb preservation is the overall management aim. Recent advances in orthopaedic, vascular and plastic surgical management have seen a move towards attempting limb salvage, where amputation would previously have been performed despite significant debate regarding criteria for early amputation versus limb salvage. ${ }^{140}$ The multiple operations associated with limb salvage attempts can be associated with poor quality of life during the years that follow the initial injury, and outcomes of salvage of severely injured legs, have been thought to be worse than for early amputation. $^{51}$ Publications from the Lower Extremity Amputation versus Preservation (LEAP) study group have, however, suggested that outcomes are similar, although poor, for both approaches. ${ }^{52} 53$ The patients of the LEAP study were similar to those eligible for the HOLLT study. Another major topic of debate regarding management of severe open fractures has been the need for very early soft tissue coverage with, if necessary, free tissue transfer. ${ }^{8}$ Early microsurgical reconstruction of soft tissue deficits has been promoted for 20 years as a means of reducing late complications of non-union and infection. ${ }^{54}{ }^{55}$ While these techniques have been a major advance, and failure rates are now very low in experienced centres, the procedures are complex and leave donor site morbidity. Recent publications suggest that with adequate surgical debridement and vacuum dressings, the proportion of cases which need to be managed with free soft tissue transfer can be reduced without compromising outcomes. ${ }^{56}{ }^{57}$ If $\mathrm{HBO}$ can reduce oedema and prevent necrosis and infection, then soft tissue closure should be easier, and there may be an increased interval for optimal scheduling of major orthopaedic and plastic surgery procedures.

The longer term outcome data for both the injury and for the patient in functional terms is of particular interest. The follow-up of patients for 2 years postinjury provides the opportunity to evaluate these, as well as assessing the cost-effectiveness of the intervention.

An inevitable criticism of this study will be that it is not 'double blind'. Our group, and others, have demonstrated that double blind studies are feasible, using a 'sham' pressurisation without oxygen as a control group treatment that effectively blinds participants and nonhyperbaric clinicians to the intervention received. We did use such a sham treatment in the pilot study that preceded the HOLLT trial, but chose not to use it in this study, but rather to blind the surgeon conducting the initial surgery, and the outcome assessors and adjudicators of all measures that have a subjective component, such as scoring radiological union, and adjudicating whether infection has occurred when indicators are unclear. Provision of sham hyperbaric treatments for acute patients is logistically very time consuming and resource intensive, and the HOLLT collaborator group would not have been able to undertake this study had we selected a double blind design. Further to this, however, we felt that sham hyperbaric treatments for acutely injured patients are not therapeutically neutral. While the increase in oxygenation delivered is minimal, and the consensus opinion is that minimal pressurisation is physiologically very near neutral, the conduct of a sham hyperbaric session requires control patients to be transported away from their normal ward environment to the hyperbaric chamber for a period in excess of $2 \mathrm{~h}$. For this to happen twice a day in the first few days after major trauma is likely to increase the risk of breakdowns in continuity of surgical care, and the delivery of $\mathrm{HBO}$ can interfere with the optimal timing of the many other assessments, investigations and interventions required. The trials design attraction of providing a sham treatment was, we felt, offset by the downside of the control group receiving an intervention (transport away from the normal ward) that carried at least some risk of a negative therapeutic effect.

When an RCT is conducted with due diligence, the result, whether positive or negative, provides an important opportunity to advance knowledge and to improve patient care. ${ }^{58}$ If $\mathrm{HBO}$ improves outcomes, then this would be significant, given the high complication rates found in this group of patients. It would also have major impacts on trauma systems design, as major limb trauma would be best managed at trauma centres with hyperbaric facilities. We believe our study design and methodology will ensure that our results are seen as valid and sufficient basis for changes to clinical practice and systems if these are indicated by our findings. Should HBO prove to not have a significant effect, or should it be associated with poor outcomes, then this will also be a critical finding that will enable us to develop recommendations preventing inappropriate use of $\mathrm{HBO}$.

\section{Author affiliations}

${ }^{1}$ Hyperbaric Service, Department of Intensive Care \& Hyperbaric Medicine, The Alfred Hospital, Melbourne, Victoria, Australia

${ }^{2}$ Department of Epidemiology and Preventive Medicine, School of Public Health and Preventive Medicine, Monash University, Melbourne, Victoria, Australia

${ }^{3}$ Department of Physiology and Pharmacology, Section for Anesthesiology and Intensive Care Medicine, Karolinska Institutet at Karolinska University Hospital, Stockholm, Sweden

${ }^{4}$ Department of Molecular Medicine and Surgery, Karolinska Institutet at Karolinska University Hospital, Stockholm, Sweden

${ }^{5}$ Ostrava City Hospital, Ostrava, Czech Republic 
${ }^{6}$ University of Ostrava, Ostrava-Zabreh, Czech Republic

${ }^{7}$ Royal Hobart Hospital, Hobart, Tasmania, Australia

${ }^{8}$ Hospital Pedro Hispano, Rua Dr. Eduardo Torres, Matosinhos, Portugal

${ }^{9}$ Department of Orthopaedic Surgery, The Alfred Hospital, Melbourne,

Victoria, Australia

${ }^{10}$ Department of Anaesthesia and Perioperative Medicine, The Alfred Hospital and Monash University, Melbourne, Victoria, Australia

Acknowledgements The participation of each collaborating centre has only been possible as a result of individual clinical leadership and the willingness of many staff at each site to contribute to an international clinical research effort. Ms Cate Venturoni was Research Nurse for the original Pilot Study conducted at The Alfred Hospital, and with OW was central to establishing many practical aspects of this study and to the detailed design of the data collection instruments. Dr Armin Kemmer and his colleagues at BGU Murnau, Germany, shared their extensive practical experience and enthusiasm for the use of HBOT in acute trauma care, and strongly supported us in designing and establishing this study. An International Steering Group helped establish this study, advising on key design features and seeking potential collaborators: A/Prof Folke Lind (Stockholm, Sweden), Prof Lin Weaver (Salt Lake City, USA), Prof Daniel Mathieu (Lille, France), A/Prof Michael Bennett (Sydney, Australia), Dr Armin Kemmer (Murnau, Germany) and Prof Thomas Kossmann (Melbourne, Australia). Alfred Hospital and Monash University orthopaedic surgeons Mr Russell Miller, A/Prof Elton Edwards and Mr Max Esser provided valuable input during the data set design phase.

Contributors ILM, PM, OW, PC and FL all made substantial contributions to the conception and design of the study. RM undertook pilot scoring and provided refinement of outcome measure adjudication methods. FL, K-AJ, MH, DS and TF led the establishment of the major collaborating sites. FL acts as European Coordinator. ILM and RAM drafted the manuscript. All authors provided critical review of the article. All authors approved the final manuscript.

Funding This work was supported by the Victorian Trauma Foundation Pty Ltd (Grant NV12) and the National Health and Medical Research Council (Project Grant 490966). The participation of the Royal Hobart Hospital was enabled by a grant from the Tasmanian Motor Accidents Insurance Board. The funders had no role in the design of the study and have no involvement or requirements regarding data collection, analysis or preparation of the manuscript. The funders have no role in any decisions regarding publication.

Competing interests None declared.

Ethics approval Ethics approval was given by The Alfred Health Human Ethics Committee (206/04) and the Monash University Human Research Ethics Committee (CF07/4208). Approval was also obtained from the institutional research ethics committee at each participating site.

Provenance and peer review Not commissioned; peer reviewed for ethical and funding approval prior to submission.

List of participating sites Australia: The Alfred, Melbourne: Ian Millar, Cate Venturoni, Virginia Cable, Anniki Hill; Royal Hobart Hospital, Hobart: David Smart, Susannah Sherlock, Bebe Brown. Europe: Karolinska University Hospital, Stockholm, Sweden: Folke Lind, Karl-Åke Jansson; Ostrava City Hospital Ostrava, Czech Republic: Michal Hajek, Aleš Zatloukal, Jan Vrchovecky; Faculty Hospital of Ostrava, Ostrava, Czech Republic: Jan Stránský, Roman Madeja; Hospital Pedro Hispano, Matosinhos, Portugal: Tiago Fernandes, Oscar Comacho; Policlinico Umberto 1, Rome, Italy: Monica Rocco; Univ. Klinik f. Unfallchirurgie, Graz, Austria: Herwig Hofer, Beatrice Ratzenhofer-Komenda. India: Indraprastha Apollo Hospital, New Delhi: Tarun Sahni. USA: John Muir Trauma Center, Walnut Creek, California: Peter Benson. South America: Hospital del Trabajador, Santiago, Chile: Tania Capote, Luis Acuña.

Open Access This is an Open Access article distributed in accordance with the Creative Commons Attribution Non Commercial (CC BY-NC 4.0) license, which permits others to distribute, remix, adapt, build upon this work noncommercially, and license their derivative works on different terms, provided the original work is properly cited and the use is non-commercial. See: http:// creativecommons.org/licenses/by-nc/4.0/

\section{REFERENCES}

1. Bondurant FJ, Cotler HB, Buckle R, et al. The medical and economic impact of severely injured lower extremities. J Traumatol 1988;28:1270-3.

2. Urquhart DM, Edwards ER, Graves SE, et al. Characterisation of orthopaedic trauma admitted to adult Level 1 Trauma Centres. Injury 2006;37:120-7.

3. Bradley C, Harrison J. Descriptive epidemiology of traumatic fractures in Australia. Injury research and statistics series. Adelaide: Australian Institute of Health and Welfare, 2004.

4. Court-Brown CM, Rimmer S, Prakash U, et al. The epidemiology of open long bone fractures. Injury 1998;29:529-34.

5. Gibbons CL. Tibial shaft fractures. In: Fairbank JCT, Pynsent PB, Carr A, eds. Classification of musculoskeletal trauma. Oxford: Butterworth-Heinemann, 1999, pp 241-53.

6. Weiss RJ, Montgomery SM, Ehlin A, et al. Decreasing incidence of tibial shaft fractures between 1998 and 2004: information based on 10,627 Swedish inpatients. Acta Orthop 2008;79:526-33.

7. Court-Brown CM, Caesar B. The Epidemiology of Fractures. In: Rockwood CA, Green DP, Bucholz RW, eds. Rockwood and Green's fractures in adults. 7th edn. Lippincott Williams \& Wilkins, 2010:53-8.

8. Breugem CC, Strackee SD. Is there evidence-based guidance for timing of soft tissue coverage of grade III B tibia fractures? Int J Low Extrem Wounds 2006;5:261-70.

9. Harris AM, Althausen PL, Kellam J, et al. Complications following limb-threatening lower extremity trauma. J Orthop Trauma 2009;23:1-6.

10. MacKenzie EJ, Jones AS, Bosse MJ, et al. Health-care costs associated with amputation or reconstruction of a limb-threatening injury. J Bone Joint Surg Am 2007;89:1685-92.

11. Butcher JL, MacKenzie EJ, Cushing B, et al. Long-term outcomes after lower extremity trauma. J Trauma 1996;41:4-9.

12. MacKenzie EJ, Burgess AR, McAndrew MP, et al. Patient-oriented functional outcome after unilateral lower extremity fracture. J Orthop Trauma 1993;7:393-401.

13. MacKenzie EJ, Cushing BM, Jurkovich GJ, et al. Physical impairment and functional outcomes six months after severe lower extremity fractures. J Trauma 1993;34:528-38; discussion 538-529.

14. Urquhart DM, Williamson OD, Gabbe BJ, et al. Outcomes of patients with orthopaedic trauma admitted to level 1 trauma centres. Aust $N$ Z J Surg 2006;76:600-6.

15. Niinikoski J. Which role for HBOT to improve tissue salvage after acute muscul-skeletal trauma? International joint meeting on hyperbaric and underwater medicine. Bologna: Grafica Victoria, 1996.

16. Strauss M. Crush injury, compartment syndrome and other acute traumatic ischaemias. In: Whelan K ed. Hyperbaric medicine practice. Flagstaff: Best Publishing, 2004:753-78.

17. Kemmer A. Crush injury and other acute ischaemias. In: Mathieu D, ed. Handbook on hyperbaric medicine. Dordecht: Springer, 2006:305-28.

18. Garcia-Covarrubias L, McSwain NEJ, Van Meter K, et al. Adjuvant hyperbaric oxygen therapy in the management of crush injury and traumatic ischemia: an evidence-based approach. Am Surg 2005;71:144-51.

19. Friedman HI, Fitzmaurice M, Lefaivre JF, et al. An evidence-based appraisal of the use of hyperbaric oxygen on flaps and grafts. Plast Reconstr Surg 2006;117(7 Suppl):175S-90S; discussion 191S-192S.

20. Goldman RJ. Hyperbaric oxygen therapy for wound healing and limb salvage: a systematic review. PM R 2009;1:471-89.

21. Wang C, Schwaitzberg S, Berliner E, et al. Hyperbaric oxygen for treating wounds: a systematic review of the literature. Arch Surg 2003;138:272-9; discussion 280.

22. Dauwe PB, Pulikkottil BJ, Lavery L, et al. Does hyperbaric oxygen therapy work in facilitating acute wound healing: a systematic review. Plast Reconstr Surg 2014;133:208e-15e.

23. Hsu RW, Hsu WH, Tai CL, et al. Effect of hyperbaric oxygen therapy on patellar tendinopathy in a rabbit model. $J$ Trauma 2004;57:1060-4.

24. Ishii $\mathrm{Y}$, Miyanaga $\mathrm{Y}$, Shimojo $\mathrm{H}$, et al. Effects of hyperbaric oxygen on procollagen messenger RNA levels and collagen synthesis in the healing of rat tendon laceration. Tissue Eng 1999;5:279-86.

25. Ishii $Y$, Ushida T, Tateishi T, et al. Effects of different exposures of hyperbaric oxygen on ligament healing in rats. J Orthop Res 2002;20:353-6.

26. Kawashima M, Tamura H, Nagayoshi I, et al. Hyperbaric oxygen therapy in orthopedic conditions. Undersea Hyperb Med 2004;31:155-62. 
27. Yablon IG, Cruess RL. The effect of hyperbaric oxygen on fracture healing in rats. J Trauma 1968;8:186-202.

28. Zamboni WA, Brown RE, Roth AC, et al. Functional evaluation of peripheral-nerve repair and the effect of hyperbaric oxygen. $J$ Reconstr Microsurg 1995;11:27-9; discussion 29-30.

29. Zhao DW. Therapeutic effect of hyperbaric oxygen on recovery of surgically repaired peripheral nerve injury. Zhonghua Wai Ke Za Zhi 1991;29:118-20, 143.

30. Zamboni WA. The microcirculation and ischaemia-reperfusion: basic mechanism of hyperbaric oxygen. In: Whelan $\mathrm{K}$ ed. Hyperbaric medicine practice. Flagstaff: Best Publishing, 2004:779-94.

31. Strauss $M$. Crush injuries and skeletal compartment muscle compartment syndrome. Feldmeier JJ, ed. In: Hyperbaric oxygen committee report. Undersea and Hyperbaric Medicine Society, 2003:27-34

32. Lindstrom T, Gullichsen E, Lertola K, et al. Effects of hyperbaric oxygen therapy on perfusion parameters and transcutaneous oxygen measurements in patients with intramedullary nailed tibial shaft fractures. Undersea Hyperb Med 1998;25:87-91.

33. Bouachour G, Cronier P, Gouello JP, et al. Hyperbaric oxygen therapy in the management of crush injuries: a randomized doubleblind placebo-controlled clinical trial. J Trauma 1996;41:333-9.

34. Fischer MD, Gustilo RB, Varecka TF. The timing of flap coverage, bone-grafting, and intramedullary nailing in patients who have a fracture of the tibial shaft with extensive soft-tissue injury. $J$ Bone Joint Surg Am 1991;73:1316-22.

35. Gustilo RB, Anderson JT. Prevention of infection in the treatment of one thousand and twenty-five open fractures of long bones: retrospective and prospective analyses. J Bone Joint Surg Am 1976;58:453-8.

36. Whittle AP, Russell TA, Taylor JC, et al. Treatment of open fractures of the tibial shaft with the use of interlocking nailing without reaming. $J$ Bone Joint Surg Am 1992;74:1162-71.

37. Bosse MJ, MacKenzie EJ, Kellam JF, et al. A prospective evaluation of the clinical utility of the lower-extremity injury-severity scores. $J$ Bone Joint Surg Am 2001;83-A:3-14.

38. Caudle RJ, Stern PJ. Severe open fractures of the tibia. $J$ Bone Joint Surg Am 1987:69:801-7.

39. Gustilo RB, Mendoza RM, Williams DN. Problems in the management of type III (severe) open fractures: a new classification of type III open fractures. J Trauma 1984;24:742-6.

40. Lange $\mathrm{RH}$. Limb reconstruction versus amputation decision making in massive lower extremity trauma. Clin Orthop Relat Res 1989:92-9.

41. MacKenzie EJ, Bosse MJ, Kellam JF, et al. Characterization of patients with high-energy lower extremity trauma. J Orthop Trauma 2000;14:455-66.

42. Tarkin IS, Clare MP, Marcantonio HC, et al. An update on the management of high-energy pilon fractures. Injury 2008;39:142-54.
43. CDC. Surgical site infection manual. National Healthcare Safety Network, 2014:11-12.

44. National Health and Medical Research Council (Australia). Australian Research Council. Australian vice-chancellors' committee: national statement on ethical conduct in human research. Canberra: National Health and Medical Research Council, 2007.

45. TGA. Note for guidance on good clinical practice (CPMP/ICH/135/ 95)—annotated with TGA comments. DSEB, 2000.

46. Freedman B. Equipoise and the ethics of clinical research. $N$ Engl $J$ Med 1987;317:141-5

47. Bennett MH, Stanford RE, Turner R. Hyperbaric oxygen therapy for promoting fracture healing and treating fracture nonunion. Cochrane Database Syst Rev 2012;11:CD004712.

48. Eskes $\mathrm{A}$, Vermeulen $\mathrm{H}$, Lucas $\mathrm{C}$, et al. Hyperbaric oxygen therapy for treating acute surgical and traumatic wounds. Cochrane Database Syst Rev 2013;12:CD008059.

49. Pape HC, Grimme K, Van Griensven M, et al. Impact of intramedullary instrumentation versus damage control for femoral fractures on immunoinflammatory parameters: prospective randomized analysis by the EPOFF Study Group. J Trauma 2003;55:7-13.

50. Weitz-Marshall AD, Bosse MJ. Timing of closure of open fractures. J Am Acad Orthop Surg 2002;10:379-84.

51. Knopp W, Kugler J, Reckert P, et al. The determinants of the quality of life after a type-III open tibial fracture. The results of a multicenter study. Chirurg 1997;68:1156-61; discussion 1162.

52. Bosse MJ, MacKenzie EJ, Kellam JF, et al. An analysis of outcomes of reconstruction or amputation after leg-threatening injuries. $N$ Engl J Med 2002;347:1924-31.

53. McCarthy ML, MacKenzie EJ, Edwin D, et al. Psychological distress associated with severe lower-limb injury. J Bone Joint Surg Am 2003;85-A:1689-97.

54. Godina M. Early microsurgical reconstruction of complex trauma of the extremities. Plast Reconstr Surg 1986;78:285-92.

55. Gopal S, Majumder S, Batchelor AG, et al. Fix and flap: the radica orthopaedic and plastic treatment of severe open fractures of the tibia. J Bone Joint Surg Br 2000;82:959-66.

56. Parrett BM, Matros E, Pribaz JJ, et al. Lower extremity trauma: trends in the management of soft-tissue reconstruction of open tibia-fibula fractures. Plast Reconstr Surg 2006;117:1315-22; discussion 1323-1314.

57. Verhelle $\mathrm{N}$, Vranckx J, Van den Hof B, et al. Bone exposure in the leg: is a free muscle flap mandatory? Plast Reconstr Surg 2005;116:170-7; discussion 178-181.

58. Garg AX, Greene T, Levin NW. A well-conducted randomized trial that establishes no benefit of therapy is an important medical advance. Nephrol Dial Transplant 2008;23:52-5. 